\title{
Pathogenesis of thyroid-associated ophthalmopathy: does autoimmunity against calsequestrin and collagen XIII play a role?
}

This article was published in the following Dove Press journal:

Clinical Ophthalmology

29 April 2010

Number of times this article has been viewed

\author{
Hooshang Lahooti \\ Kishan R Parmar \\ Jack R Wall \\ The Department of Medicine, \\ University of Sydney, Nepean \\ Clinical School, Penrith, NSW, \\ Australia
}

Correspondence: Jack R Wall University of Sydney, Nepean Clinical School,

Nepean Hospital, PO Box 63, Penrith NSW 275I, Australia

Tel +6I (02) 473426 I3

Fax+6I (02) 47342614

Email jackw@med.usyd.edu.au

\begin{abstract}
Thyroid-associated ophthalmopathy (TAO), or thyroid eye disease, is a complex inflammatory disorder of the eye that, as its name implies, is associated with thyroid disease. TAO can be divided into three subtypes: ocular myopathy, congestive myopathy and mixed congestive and myopathic ophthalmopathy. Although the precise pathophysiology of TAO remains unclear it is likely to reflect an autoimmune reaction involving sensitized T-cells and autoantibodies directed against a thyroid and orbital tissue shared antigen. One well studied candidate in this immune reaction is the thyroid-stimulating hormone receptor (TSH-r), expressed in the orbital fibroblast and pre adipocyte. In our studies of TAO, we have investigated the nature and significance of antibodies targeting other eye muscle and orbital connective tissue (OCT) antigens. Our findings suggest that autoimmunity against the eye muscle antigen calsequestrin and the OCT antigen collagen XIII plays a role in the pathogenesis of TAO. We propose that ocular myopathy and chronic eyelid retraction are due to autoimmunity against skeletal muscle calsequestrin in the extraocular and eyelid muscles, respectively. This may be initiated in the thyroid where calsequestrin expression is upregulated, possibly due to a stimulatory effect of TSH-r antibodies. We also propose that congestive ophthalmopathy results from a reaction against the TSH-r or collagen XIII in orbital fibroblast cell membranes. Further insight into the role of eye muscle and OCT antigens in the pathogenesis of TAO may allow for the development of new therapies to treat the eye disorder and reduce patient morbidity.
\end{abstract}

Keywords: thyroid-associated ophthalmopathy, autoimmunity, t calsequestrin, collagen XIII

\section{Introduction}

Ophthalmopathy is a common manifestation of Graves' disease. The association between "poppy eyes" and goitre was described as early as 1,000 AD by the Persian physician and philosopher Avicenna. ${ }^{1}$ Although ophthalmopathy is most commonly seen in patients with Graves' hyperthyroidism, in whom it is called Graves' ophthalmopathy (GO). ${ }^{2,3}$ It is also present in a small proportion of patients with transient (subacute and silent) thyroiditis., ${ }^{4,5}$ Mild eye changes, particularly upper eyelid retraction (UER), are present in about a third of patients with progressive (Hashimoto's) thyroiditis. ${ }^{6}$ Ophthalmopathy also occurs in the apparent absence of thyroid autoimmunity in $10 \%$ of patients, but is presumed to be the same disorder, ie, an autoimmune ophthalmitis. The most appropriate name may be thyroid-associated ophthalmopathy (TAO), or thyroid orbitopathy. Here, we use the general term TAO for the spectrum of eye changes in patients with thyroid disease and GO when we refer to the ophthalmopathy associated with Graves' hyperthyroidism.

The precise pathophysiologic mechanisms underlying the development of TAO and the explanation for its link with the thyroid are incompletely understood. 
An understanding of these mechanisms is however essential due to the considerable morbidity associated with $\mathrm{TAO}^{7,8}$ and the need for specific and safe therapies. The general consensus is that TAO is likely to reflect an autoimmune reaction during which sensitized T-cells and autoantibodies are directed against a thyroid and orbital tissue shared antigen such as the thyroid-stimulating hormone receptor (TSH-r) which is expressed in the thyroid, OCT and fat and, possibly, the extraocular muscle fiber (Box 1). ${ }^{9}$

However, recent research, mainly from the authors' laboratory over the last decade, has suggested a possible important role for autoimmunity against other OCT and eye muscle antigens (Figures 1 and 2). The main antibodies proposed are those targeting i) the flavoprotein (Fp) subunit of mitochondrial succinate dehydrogenase; ii) G2s, shown to be a fragment of the FOX-P1 transcription factor; iii) the calcium binding protein calsequestrin; and iv) collagen XIII, a connective tissue antigen expressed in the orbital fibroblast cell membranes.

In this review, we attempt to summarize recent advances in the pathogenesis of TAO with a particular emphasis on a possible role of autoimmunity against calsequestrin and collagen XIII. In doing so, we review the TSH-r hypothesis and attempt to provide a unified hypothesis that explains all the features of TAO. We will also outline possible future directions for research in this developing field.

\section{Classification of TAO}

We propose that there are three main subtypes of TAO: congestive ophthalmopathy, ocular myopathy and mixed congestive and myopathic ophthalmopathy (Table 1). Congestive ophthalmopathy is characterized by inflammation of the OCT, with relative sparing of the extra ocular muscles, and manifests with clinical features of eye swelling,

1956 LATS is the cause of Graves' hyperthyroidism (Adams and Purvis, New Zealand)

1956 LATS is an immunoglobulin G (IgG) antibody against TSH-r

1964 TSH-r antibody (measured as LATS) crosses the placenta and causes neonatal hyperthyroidism, but ophthalmopathy not observed

1973 Graves' ophathalmopathy is associated with higher LATS concencentrations in blood

1986 Cytotoxic antibodies act against eye muscle cells in Graves' ophthalmopathy

1988 "Thyroid-associated ophthalmopathy" suggested as the best name for the eye disorder, which occasionally occurs in patients with Hashimoto's thyroiditis

1988 55-, 64- and 95-kDa eye-muscle membrane proteins are recognized by serum autoantibodies in TAO

1993 TSH-r is expressed in retro-orbital tissue; corresponding serum autoantibodies are postulated to be the cause of the ophthalmopathy

1998 The $64-k D a$ protein is the flavoprotein subunit of mitochondrial succinate dehydrogenase

1999 Orbital tissue membrane antigens are recognized by sensitized T-lymphocytes in the blood of people with TAO

2000 Four subtypes of TAO are categorized: ocular myopathy, congestive ophthalmopathy, mixed disease and chronic eyelid disease

2000 G2s (a fragment of FOXP1 transcription factor), sarcalumenin and calsequestrin are identified as eye-muscle antigens in TAO

2005 Collagen type XIII in cell membranes of orbital fibrolasts is a target antigen in the congestive ophthalmopathy subtype of TAO

2005 Anticalsequestrin antibodies are sensitive and specific markers of the ocular myopathy subtype of TAO

Note: LATS = long-acting thyroid stimulator, TSH-r = thyroid-stimulating hormone receptor.

Box I Timeline of discoveries related to thyroid-associated ophthalmopathy (TAO). Reproduced with the permission from Tani J,Wall JR. Autoimmunity against eye-muscle antigens may explain thyroid-associated ophthalmopathy. CMAJ. 2006;175(3):239. ${ }^{10}$ Copyright ( 2006 Canadian Medical Association 
Table I Thyroid associated ophthalmology (TAO) subtypes, clinical features and candidate autoantibodies

\begin{tabular}{lll}
\hline TAO subtype & Main clinical features & Candidate autoantigens \\
\hline Ocular myopathy & Diplopia & Calsequestrin \\
& EOM dysfunction & Flavoprotein \\
& Exophthalmos & TSH-r \\
Congestive ophthalmopathy & Watery, gritty eyes & Collagen XIII \\
& Periorbital edema & \\
Mixed congestive and myopathic & Conjunctival injection/chemosis & All of the above \\
ophthalmopathy & Exophthalmos & Congestive and myopathic \\
\hline
\end{tabular}

${ }^{\mathrm{a}} \mathrm{G} 2 \mathrm{~s}$ is a fragment of the FOX-PI transcription factor.

Abbreviations: EOM, extra ocular muscle; TSH-r, thyroid-stimulating hormone receptor.

conjunctival injection, chemosis, watery or gritty eyes and exophthalmos. In contrast, ocular myopathy is characterized by inflammation and swelling of the extraocular muscles and manifests as eye muscle dysfunction and diplopia and occasionally, painful eye movements. Although congestive and myopathic features can occur in isolation, mixed congestive and myopathic ophthalmopathy is the most common presentation of TAO, occurring in approximately $60 \%$ of TAO patients. ${ }^{10}$ In patients with Hashimoto's thyroiditis, UER and lag are often the only features of an ophthalmopathy except for and mild proptosis. ${ }^{6}$

\section{Pathogenesis of TAO TSH-r hypothesis}

TAO has been described as a limited multi system autoimmune disorder involving antigens in the OCT, eye muscle fiber, the lacrimal gland, and human harderian gland equivalent and the thyroid gland. ${ }^{11}$ The overall evidence to suggest that TAO is an autoimmune disorder is strong and no-one seriously doubts this. The uncertainty which makes TAO a controversial disorder is the identity of the antigens involved, whether the eye muscles or OCT is the primary target tissue in the orbit and how the ophthalmopathy is linked to thyroid autoimmunity. As previously mentioned, the general hypothesis has been that this association is due to cross reactivity, ie, antibodies and T cells targeting proteins expressed in both the thyroid and eye. A popular theory is that the primary reaction may involve antibodies targeting the TSH-r in the OCT which leads to orbital inflammation, manifest as orbital fibroblast stimulation, collagen and glycosaminoglycans (GAGS) production and associated congestive eye features. ${ }^{12}$ According to this theory, ocular myopathy is due to secondary, ischemic, damage to the eye muscles following primary OCT inflammation. Certainly, the TSH-r is a logical candidate antigen as it is expressed in orbital preadipocytes and fibroblasts as well as the thyroid gland. ${ }^{12,13}$ Its expression in other tissues such as fat and connective tissue may also help to explain the development of pretibial myxedema and thyroid acropachy in some patients with Graves' disease. ${ }^{14,15}$ Although not shown in all reports, some studies have shown that TSH-r antibody levels correlate directly with clinical features of $\mathrm{GO}^{7}$ further implicating its role in the pathogenesis of TAO.

Although the evidence provides strong overall support for a role of the TSH-r in the pathogenesis of TAO, whether TSH-r antibodies are really linked to ophthalmopathy in all cases of TAO remains unclear. TSH-r antibodies cannot explain the development of ophthalmopathy in patients in whom the eye changes occur many years after the development of Graves' hyperthyroidism. Furthermore, the TSH-r is also expressed in fibroblasts and adipocytes at sites such as the abdominal wall which are unaffected in Graves' disease patients. ${ }^{16}$ In patients with Hashimoto's thyroiditis, eye changes occur in the presumed absence of TSH-r antibody production. Finally, newborns with thyrotoxicosis do not have ophthalmopathy, even when the mother has eye signs. These findings suggest that a specific link between thyroid autoimmunity/the TSH-r and ophthalmopathy has not been established in all situations. ${ }^{17}$

\section{Eye muscle auto antigens}

Potential eye muscle antigens in TAO were first reported in the 1980 s by Kodama et a ${ }^{18}$ and later by others. ${ }^{19-23}$ The initial antigens were detected according to their molecular weight by western blotting and the most consistently reported eye muscle membrane proteins recognized by serum autoantibodies in TAO patients were proteins of 55 and $64 \mathrm{kDa} .{ }^{24} \mathrm{The}$ $55 \mathrm{kDa}$ protein, later called $\mathrm{G} 2 \mathrm{~s},{ }^{25}$ was recognized to be a fragment of the FOX-P1 transcription factor \{human genome project\}. It was also demonstrated that there were in fact three 
$64 \mathrm{kDa}$ proteins; Fp, the 1D protein and calsequestrin. ${ }^{26}$ In our earlier studies, using a crude preparation of calsequestrin prepared from rabbit heart muscle and by measuring corresponding calsequestrin antibodies by western blot analysis we found a modest relationship between anti-calsequestrin antibodies and ophthalmopathy. Anti-calsequestrin antibodies were detected in $40 \%$ of patients with clinically active TAO but only in $5 \%$ of normal subjects. ${ }^{27}$ In a separate study we demonstrated that anti-G2s antibodies were also associated with active ophthalmopathy in patients with Graves' hyperthyroidism, being detected in 54\% of patients with active TAO compared to $33 \%$ of those with inactive disease. Anti-G2s antibody detection was shown to precede the development of congestive ophthalmopathy in 7 out of 8 patients and ocular myopathy in 6 out of 7 patients. ${ }^{28}$ In patients with Graves' hyperthyroidism, early studies also highlighted the role of Fp as a potential marker of eye muscle inflammation. Anti-Fp antibodies were detected in $66 \%$ of patients with ophthalmopathy and eye muscle involvement and in $32 \%$ of patients with congestive ophthalmopathy. ${ }^{28}$ Fp antibody positivity was also shown to precede the development of ocular myopathy, but not congestive ophthalmopathy, in 5 out of 6 patients. ${ }^{28}$

Although these initial studies seemed to demonstrate that antibodies against Fp and G2s were closely linked to the eye muscle component of ophthalmopathy neither are specific to the eye muscle and both antigens are expressed in tissues not normally involved in Graves' disease. ${ }^{3,29}$ Moreover, Fp and G2s are both intracellular proteins and would not encounter T-cells or antibodies until the eye muscle fiber cell membrane had been breached. It turns out that anti-G2s antibodies are not specific to TAO, being detected in 5\% to $10 \%$ of healthy subjects and in a few patients with thyroid autoimmunity without ophthalmopathy. ${ }^{25,29,30}$ The presence of both antibodies have also been demonstrated in $60 \%$ of patients with non-specific orbital inflammation ${ }^{31}$ and anti-Fp antibodies in $50 \%$ of patients with ocular myasthenia gravis. Taking all the evidence into account, it would appear that antibodies against G2s and Fp are nonspecific markers of eye muscle damage.

\section{Roles of autoimmunity against calsequestrin and collagen XIII}

When Porter et $\mathrm{al}^{32}$ showed that calsequestrin was expressed 4.8 times more in eye muscle compared to other skeletal muscle, thus offering an explanation for the orbital specificity of skeletal muscle inflammation in Graves' disease, we decided to re visit its possible role in the pathogenesis of TAO.
Calsequestrin seemed a good candidate as it was shown to be distributed throughout the cell during the myotube stage of differentiation, where it could be seen by antibodies and T-cells (Beard N, pers comm). At that time we were also interested in the connective tissue antigen collagen XIII because serum antibodies against collagen XIII were shown to be good markers of ophthalmopathy in patients with active ophthalmopathy. ${ }^{33}$

In our initial studies we reinvestigated the prevalence of anti-calsequestrin antibodies in a large group of patients with thyroid autoimmunity with and without ophthalmopathy and in healthy subjects. ${ }^{34}$ These studies were performed using enzyme-linked immunosorbent assay (ELISA) incorporating highly purified rabbit skeletal muscle calsequestrin which shares 97\% homology with human calsequestrin. We demonstrated that calsequestrin antibodies were good markers of ophthalmopathy, in particular of the ocular myopathy subtype of TAO in patients with Graves' hyperthyroidism, being generally negative in patients with Hashimoto's thyroiditis without eye signs, multi nodular goitre and in healthy control subjects. ${ }^{34}$ We also demonstrated that anticalsequestrin antibodies correlated with the onset of ocular myopathy in 6 out of 11 patients with GO. ${ }^{34}$ These findings were important as they were the first to highlight the potential role of calsequestrin antibodies in the differential diagnosis of orbital inflammation and as markers to assess the efficacy of therapies for ophthalmopathy.

In light of these findings, and to further characterize the role of eye muscle and collagen XIII antigens in the pathogenesis of TAO, we conducted a study in which we determined the prevalence and levels of antibodies against calsequestrin, collagen XIII, Fp and G2s in the same study cohort, using ELISA. The prevalences of calsequestrin and collagen XIII antibodies, and to a lesser extent Fp antibodies, were significantly greater in patients with GO compared to healthy controls and in GO patients compared to those with Graves' hyperthyroidism patients without ophthalmology, which was not the case for antibodies targeting G2s. Finally, positive antibody reactivity against calsequestrin and collagen XIII was not observed in any of the healthy controls, while Fp and G2s antibodies were found in $14.3 \%$ and $4.8 \%$ respectively, of controls. ${ }^{35}$

To further characterize the mechanism for chronic eyelid disease we carried out a similar study in patients with; Graves' disease with UER as a dominant feature of their ophthalmopathy, Hashimoto's thyroiditis with UER as an isolated abnormality and non-immunologic thyroid disease associated with UER. ${ }^{36}$ We found a generally good correlation between 

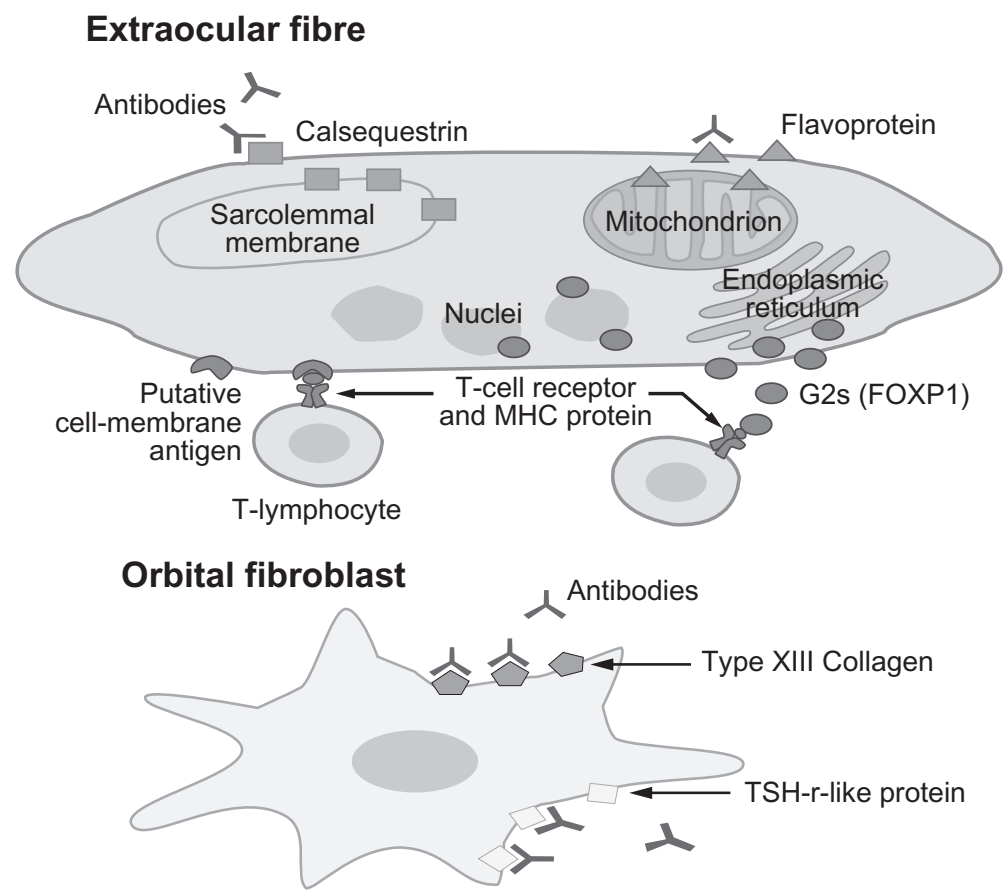

Figure I Eye muscle and orbital fibroblast autoantigens recognized by T lymphocytes or antibodies in thyroid-associated ophthalmopathy (TAO). Our working hypothesis is that the ocular myopathy subtype of TAO is initiated by T lymphocyte-mediated targeting of calsequestrin or a yet unidentified eye muscle cell membrane antigen. Serum antibodies against flavoprotein, G2s and sarcalumenin are likely to be secondary to release of the proteins following muscle fiber necrosis. Chronic eyelid disease, which is a feature of TAO or a dominant sign in patients with Hashimoto's thyroiditis, may be the result of T lymphocyte-mediated targeting of calsequestrin in the upper eyelid levator palpebrae superioris muscle. The congestive ophthalmopathy subtype of TAO is likely to result from a reaction against the TSH-r or collagen $\mathrm{XIII}$ in the fibroblast cell membranes, which leads to fibroblast stimulation and excess production of collagen and glycosaminoglycans. The different reactions shown in the figure may occur alone or in combination. Reproduced with the permission from Tani J, Wall JR. Autoimmunity against eye-muscle antigens may explain thyroid-associated ophthalmopathy. CMAJ. 2006; 175(3):239..$^{10}$ Copyright (c) 2006 Canadian Medical Association

Abbreviations: MHC, major histocompatibility complex; TSH-r, thyroid-stimulating hormone receptor.

the prevalence of antibodies targeting calsequestrin and collagen XIII and the severity of UER in these patient groups. Calsequestrin is highly expressed in the EOM ${ }^{32}$ including, presumably, the levator palpebrae superioris (LPS) muscle. Type XIII collagen, expressed in fibroblast cell membranes, is also widely expressed in the adult and developing human eye. ${ }^{36}$ Although yet to be proven by histological analysis, our findings suggest that chronic UER and lag may reflect autoimmunity against calsequestrin and collagen XIII in the LPS muscle.

\section{Ophthalmopathy in patients with thyroiditis}

Subacute thyroiditis (SAT) and silent thyroiditis (ST) are the most common causes of transient thyrotoxicosis. In both, hyperthyroidism is the result of rapidly progressive thyroid follicular cell injury and the release of large amounts of thyroid hormone into the systemic circulation. Hypothyroidism, a consequence of thyroid cell damage and the subsequent depletion of thyroid hormone stores, often follows. SAT is caused by viral induced inflammation of the thyroid gland. Sporadic ST, on the other hand, appears to be an autoimmune disease. There have been reports of the development of TSH-r positive Graves' hyperthyroidism and ophthalmopathy following SAT. ${ }^{37,38}$ However, Amato et al ${ }^{39}$ recently reported the case of a Caucasian male who developed euthyroid Graves' disease after proven SAT in the absence of TSH-r antibodies but with detectable eye muscle antibodies. This prompted us to study the prevalence of eye and eyelid signs and positive eye muscle and collagen XIII antibody tests in 11 patients with transient thyroiditis (5 with SAT and 6 with ST), 11 patients with Hashimoto's thyroiditis as chronic thyroiditis controls and in 12 age and sex-matched healthy controls. ${ }^{5}$ In summary, 5 patients with transient thyroiditis and 5 with Hashimoto's thyroiditis were found, at first visit or on follow-up, to have developed ophthalmopathy. TSH-r antibodies were found in only one patient who developed Graves' hyperthyroidism following an episode of ST. One or more eye muscle or collagen XIII antibodies were detected in 7 patients with transient thyroiditis and in 7 with Hashimoto's thyroiditis, and antibody levels correlated generally with eye signs. Calsequestrin and $\mathrm{Fp}$ antibodies were the most frequently detected and collagen XIII antibodies were detected in only two patients with ST and one patient 


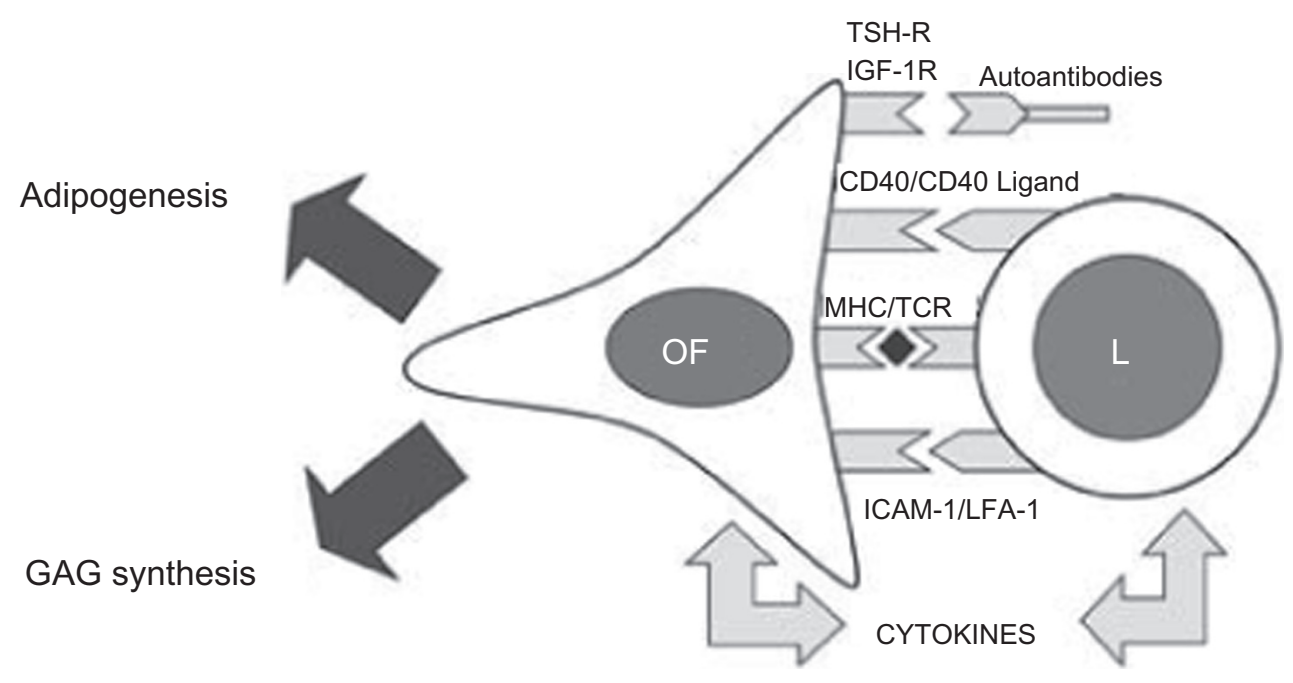

Figure 2 Orbital fibroblast activation in thyroid-associated ophthalmopathy. The main features of Graves' ophthalmopathy include the enlargement of eye muscles and the expansion of the orbital fatty connective tissues. These changes result from accumulation of glycosaminoglycans (GAGs) and edema within these tissues and de novo adipogenesis. The orbital fibroblast (OF) appears to be the major effector cell in GO influencing both processes. In the active stage of disease, OF are capable of producing large amounts of GAGs following (i) stimulation by cytokines (especially Th-I cytokines), (ii) activation of CD40 by CD40 ligand and (iii) activation of insulin growth factor receptor (IGF-IR) by autoantibodies. A subset of OF, the preadipocytes, are capable of differentiating into mature adipocytes following appropriate stimulation by cytokines (IL-6) and activation of thyroid-stimulating hormone receptor (TSH-r). In addition, OF have been shown to display the immunoregulatory molecules major histocompatibility complex MHC class II (HLA-DR) and intercellular adhesion molecule-I (ICAM-I), and also are capable of secreting chemokines and cytokines which stimulate the infiltration of activated T cells into areas of inflammation. In the 'burn-out' stage of ophthalmopathy, OF participate in tissue fibrosis. Reproduced with permission from Bednarczuk T, Gopinath B, Ploski R, Wall JR. Susceptibility genes in Graves' ophthalmopathy: searching for a needle in a haystack? Clin Endocrinol (Oxf). 2007; 67(I):3-19.45 Copyright @ 2007 Wiley Blackwell.

Abbreviations: LFA, lymphocyte function-associated antigen; MHC, major histocompatibility complex.

with Hashimoto's thyroiditis. ${ }^{5}$ These findings suggest that ophthalmopathy associated with destructive thyroiditis is mainly an eye muscle disorder.

To further study the prevalence and phenotype of ophthalmopathy in patient with Hashimoto's thyroiditis, we have recently studied 91 recently diagnosed patients with Hashimoto's thyroiditis for eye and upper eyelid signs and correlated these with calsequestrin and collagen XIII antibodies. ${ }^{6}$ We showed that the prevalence of eye signs in patients with Hashimoto's thyroiditis was much greater than previously thought, being present in $34 \%$ of the patients. This observation again raises questions about the role of the TSH-r in the pathogenesis of ophthalmopathy. Because TSH-r antibodies are expected to be negative in the majority of patients with Hashimoto's thyroiditis, their ophthalmopathy cannot be explained by autoimmunity against the "TSH-r in the LPS muscle".

\section{T lymphocyte reactivity against orbital antigens}

Recent studies addressing the putative role of cell-mediated immunity in TAO have also generated important insights into the pathogenesis of this disorder. ${ }^{40}$ Immunohistological analysis has shown that TAO is characterized by the infiltration of EOM and OCT by mononuclear cells consisting predominantly of T-cells. ${ }^{41-43}$ We investigated the role of peripheral blood T cell mediated reactivity against calsequestrin, Fp and G2s in 22 patients with GO, 9 patients with Graves' hyperthyroidism without ophthalmopathy and in 16 age and sex matched healthy controls, using a standard lymphocyte proliferation assay. ${ }^{44}$ The prevalence of positive lymphocyte reactivity to calsequestrin was $58 \%$ in all patients with GO, $67 \%$ in those with active disease and in 33\% of patients with Graves' hyperthyroidism without ophthalmopathy. Positive lymphocyte reactivity to Fp and G2s however was not significantly different between patients with GO, Graves' hyperthyroidism without ophthalmopathy or healthy controls. ${ }^{44}$

The specific mechanisms responsible for $\mathrm{T}$ cell-migration into orbital tissues and autoantigen recognition and the roles of costimulatory signal activation and cytokines, are poorly understood. We postulate that the eye muscle component of TAO is initiated by T-cells targeting some cell membrane antigen, possibly calsequestrin. Thereafter, these recruited T-cells may release various cytokines that stimulate retro-orbital fibroblasts to proliferate and secrete GAGs, leading to congestive ophthalmopathy and orbital fibrosis (Figures 1 and 2).

\section{Risk factors for ophthalmopathy}

The phenotypic variation of eye signs in patients with TAO suggests that complex interactions between endogenous 
(genetic factors, increasing age, male sex) and exogenous (cigarette smoking, hyperthyroidism and hypothyroidism, radioiodine treatment) factors may influence the development and severity of ophthalmopathy ${ }^{45}$ Smoking appears to be the greatest risk factor for ophthalmopathy ${ }^{46,47}$ while the role of genetic factors in its development remains unclear. ${ }^{45}$ Recent work has focused on identifying genetic alterations associated with GO through small scale case-controlled association studies with candidate genes. Several susceptibility loci in $\mathrm{GO}$ have now been proposed which include the human leucocyte antigen (HLA, 6p21.3), cytotoxic T-lymphocyte antigen-4 (CTLA-4, 2q33), tumor necrosis factor (TNF, $6 \mathrm{p} 21.3)$, interferon- $\gamma(\mathrm{IFN}-\gamma, 12 \mathrm{q} 14)$, intracellular adhesion molecule-1 (ICAM-1, 19p13), and the thyroid stimulating hormone receptor gene (TSH-R, 14q31). The results of association studies published so far however are inconclusive because the vast majority of them are underpowered and poorly characterized..$^{45}$

\section{Microarray expression studies}

Recently, we extracted total RNA prepared from thyroid tissue of patients with Graves' hyperthyroidism with (GO) and without (GH) ophthalmopathy and tested it for expression across 20,589 genes determined using micro array gene expression analysis. ${ }^{48}$ We were looking for differences in gene expression between these two patient groups which might provide insight into the early thyroid events that lead to the development of ophthalmopathy. Of the 20,589 genes surveyed, 296 genes were identified to be differentially expressed between patients with GO and GH. Of these genes, the skeletal muscle calsequestrin gene, CASQ1, was up-regulated 4.1-fold in GO vs GH, although this was not statistically significant. Surprisingly, the cardiac calsequestrin gene, CASQ2, was the most highly up-regulated gene, 2.2-fold, in thyroid tissue from GO vs GH. The orbital antigen, Fp, was also found to be significantly up-regulated, 1.4-fold, while genes encoding collagen XIII, FOXP1/G2s, thyroglobulin, thyroid peroxidise and the TSH-r, were not differentially expressed. ${ }^{48}$

Skeletal muscle calsequestrin and cardiac muscle calsequestrin are closely related proteins that share $68.4 \%$ homology. Interestingly, like skeletal muscle calsequestrin, cardiac muscle calsequestrin is also expressed more in the eye muscle than other skeletal muscle, 2.7 -fold. ${ }^{49}$ The finding that the CASQ2 gene was the most highly upregulated gene in thyroid tissue from patients with GO is unlikely to be a chance finding. Although the CASQ1 gene was not significantly up-regulated in GO patients it is possible that one or more of the patients in GH patients "without eye signs" manifested subclinical ophthalmopathy at the time of thyroidectomy. It is clear that calsequestrin genes are not supposed to be expressed in the thyroid gland and their upregulation in the thyroid of patients with ophthalmopathy provides further support for a role of autoimmunity against calsequestrin in its pathogenesis. The next step would be immune sensitization to calsequestrin and cross-reactivity against the same protein in eye and eyelid muscles.

\section{Unifying hypothesis}

Most workers and readers accept the TSH-r hypothesis as being fact. ${ }^{50}$ However, recent developments have suggested a possible role for autoimmunity against other orbital antigens in the pathogenesis of TAO, as described above, and this deserves to be taken seriously. We postulate that while antibodies directed against the TSH-r may be the initiating event that leads to orbital inflammation, collagen XIII is also good candidate antigen in the congestive subtype of TAO as it has been shown to be expressed in the plasma membrane of orbital fibroblasts, and thus accessible to the immune system. Antibody and $\mathrm{T}$ lymphocyte reactivity against calsequestrin, or some other eye muscle cell membrane antigen, may separately and independently lead to eye and eyelid muscle inflammation and damage, manifest as diplopia and UER (Figure 1). It seems less likely that autoimmunity against the TSH-r leads to secondary eye muscle damage particularly as ocular myopathy can occur as an isolated abnormality occasionally in patients with no associated thyroid disease. Support for the theory of primary autoimmunity involving the eye muscles can be demonstrated by electron microscope examination in GO patients with relatively recent onset disease showing muscle fiber damage in the absence of OCT inflammation. $^{14}$

\section{Future directions}

Although our studies have raised the possibility that autoimmunity against calsequestrin and collagen XIII may play a role in the pathogenesis of TAO this is not proven. Larger prospective studies of patients with newly diagnosed TAO in which serum antibody levels and parameters of blood and, better, orbital $\mathrm{T}$ lymphocyte reactivity against the two antigens are correlated with clinical features of TAO and accurate measurements of extra ocular muscle volumes from orbital imaging, are needed. Patients with other skeletal muscle inflammatory and autoimmune disorders should be studied to confirm disease specificity of the serum antibodies. Other possible avenues for future research include elucidating 
the mechanisms regulating $\mathrm{T}$ lymphocyte migration into retroorbital tissue, there activation by autoantigens and the role of cytokines and co stimulatory signals. It is also important to attempt to develop an animal model in which we can further investigate the nature and significance of calsequestrin and collagen XIII in the pathogenesis of TAO.

Finally, the role of putative genetic factors in the development of ophthalmopathy remain unclear. So far, casecontrolled studies with candidate genes have identified several susceptibility loci, however important candidate genes including those encoding orbital autoantigens and genes involved in GAGs synthesis remain to be studied. Through further association studies these candidate genes should be explored in a prospective multicenter study involving a large number of patients with well defined thyroid disease with and without ophthalmopathy. Whole genome analysis for polymorphisms of the calsequestrin and collagen XIII genes may also provide information about new genes and their proteins involved in the pathogenesis of TAO which could be considered as targets for therapy.

\section{Disclosures}

The authors declare no conflicts of interest.

\section{References}

1. Nabipour I, Burger A, Moharreri MR, Azizi F. Avicenna, the first to describe thyroid-related orbitopathy. Thyroid. 2009;19(1):7-8.

2. Burch HB, Wartofsky B. Graves' ophthalmopathy: current concepts regarding pathogenesis and management. Endocr Rev. 1993;14(6):747-793.

3. Yamada M, Wu Li A, Wall J. Thyroid-associated ophthalmopathy: clinical features, pathogenesis, and management. Crit Rev Clin Lab Sci. 2000;37(6):523-549.

4. Gopinath B, Adams CL, Musselman R, Tani J, Wall JR. Antibodies against calsequestrin and type XIII collagen are good markers for chronic upper eyelid retraction. Ocul Immunol Inflamm. 2007;15(2):81-88.

5. Gopinath B, Ma G, Wall JR. Eye signs and serum eye muscle and collagen XIII antibodies in patients with transient and progressive thyroiditis. Thyroid. 2007;17(11):1123-1129.

6. Tjiang H, Lahooti H, McCorquodale T, Wall JR. Eye and eyelid abnormalities are common in patients with Hashimoto's thyroiditis. Thyroid. 2010. In Press.

7. Coulter I, Frewin S, Krassas GE, Perros P. Psychological implications of Graves' orbitopathy. Eur J Endocrinol. 2007;157(2):127-131.

8. Della Rocca RC. Thyroid-related orbitopathy: concepts and management. Facial Plast Surg. 2007;23(3):168-173.

9. Busuttil BE, Frauman AG. Extrathyroidal manifestations of Graves' disease: the thyrotropin receptor is expressed in extraocular, but not cardiac, muscle tissues. J Clin Endocrinol Metab. 2001;86(5):2315-2319.

10. Tani J, Wall JR. Autoimmunity against eye-muscle antigens may explain thyroid-associated ophthalmopathy. CMAJ. 2006;175(3):239.

11. Wall JR. Graves' disease is a multi-system autoimmune disorder in which extra ocular muscle damage and connective tissue inflammation are variable features. "In my view". Thyroid. 2002;12:35-36.

12. Bahn RS. Understanding the immunology of Graves' ophthalmopathy. Is it an autoimmune disease? Endocrinol Metab Clin North Am. 2000;29(2):287-296, vi.
13. Bahn RS. TSH receptor expression in orbital tissue and its role in the pathogenesis of Graves' ophthalmopathy. J Endocrinol Invest. 2004;27(3):216-220

14. Heufelder AE, Dutton CM, Sarka G, Donovan KA, Bahn RS. Detection of TSH receptor RNA in cultured fibroblasts from patients with Graves' ophthalmopathy and pretibial dermopathy. Thyroid. 1993;3(4):297-300.

15. Stadlmayr W, Spitzweg C, Bichlmair AM, Heufelder AE. TSH receptor transcripts and TSH receptor-like immunoreactivity in orbital and pretibial fibroblasts of patients with Graves' ophthalmopathy and pretibial myxedema. Thyroid. 1997;7(1):3-12.

16. Bell A, Gagnon A, Grunder L, Parikh SJ, Smith TJ, Sorisky A. Functional TSH receptor in human abdominal preadipocytes and orbital fibroblasts. Am J Physiol Cell Physiol. 2000;279(2):C335-C340.

17. Wall JR, Bernard N, Boucher A, et al. Pathogenesis of thyroid-associated ophthalmopathy: an autoimmune disorder of the eye muscle associated with Graves' hyperthyroidism and Hashimoto's thyroiditis. Clin Immunol Immunopathol. 1993;68(1):1-8.

18. Kodama K, Sikorska H, Bandy-Dafoe P, Bayly R, Wall JR. Demonstration of a circulating autoantibody against a soluble eye-muscle antigen in Graves' ophthalmopathy. Lancet. 1982;2(8312):1353-1356.

19. Atkinson S, Holcomb M, Kendall-Taylor P. Ophthalmopathic immunoglobulin in patients with Graves' ophthalmopathy. Lancet. 1984;2(8399):374-376.

20. Faryna M, Nauman J, Gardas A. Measurement of autoantibodies against human eye muscle plasma membranes in Graves' ophthalmopathy. Br Med J (Clin Res Ed). 1985;290(6463):191-192.

21. Kahaly G, Moncayo R, Bemetz U, Krause U, Beyer J, Pfeiffer EF. Eye muscle antibodies in endocrine exophthalmos. Clinical and serological observations. Horm Metab Res. 1989;21(3):137-141.

22. Wu YJ, Clarke EM, Shepherd P. Prevalence and significance of antibodies reactive with eye muscle membrane antigens in sera from patients with Graves' ophthalmopathy and other thyroid and non thyroid diseases. Thyroid. 1998;8(2):167-174.

23. Chang TC, Chang TJ, Huang YS, Huang KM, Su RJ, Kao SC. Identification of autoantigen recognized by autoimmune ophthalmopathy sera with immunoblotting correlated with orbital computed tomography. Clin Immunol Immunopathol. 1992;65(2):161-166.

24. Salvi M, Miller A, Wall JR. Human orbital tissue and thyroid membranes express a $64 \mathrm{kDa}$ protein which is recognized by autoantibodies in the serum of patients with thyroid-associated ophthalmopathy. FEBS Lett. 1988;232(1):135-139.

25. Gunji K, De Bellis A, Li AW, et al. Cloning and characterization of the novel thyroid and eye muscle shared protein G2s: autoantibodies against G2s are closely associated with ophthalmopathy in patients with Graves' hyperthyroidism. J Clin Endocrinol Metab. 2000;85(4):1641-1647.

26. Kubota S, Gunji K, Stolarski C, Kennerdell JS, Wall JR. Re evaluation of the prevalences of serum autoantibodies reactive with "64-kd eye muscle proteins" in patients with thyroid-associated ophthalmopathy. Thyroid. 1998;8(2):175-179.

27. Gunji K, Kubota S, Stolarski C, Wengrowicz S, Kennerdell JS, Wall JR. A $63 \mathrm{kDa}$ skeletal muscle protein associated with eye muscle inflammation in Graves' disease is identified as the calcium binding protein calsequestrin. Autoimmunity. 1999;29(1):1-9.

28. Gunji K, De Bellis A, Kubota S, et al. Serum antibodies against the flavoprotein subunit of succinate dehydrogenase are sensitive markers of eye muscle autoimmunity in patients with Graves' hyperthyroidism. J Clin Endocrinol Metab. 1999;84(4):1255-1262.

29. El-Kaissi S, Frauman AG, Wall JR. Thyroid-associated ophthalmopathy: a practical guide to classification, natural history and management. Intern Med J. 2004;34(8):482-491.

30. Kaspar M, Archibald C, Li AW, et al. Eye muscle antibodies and subtype of thyroid-associated ophthalmopathy. Thyroid. 2002;12(3):187-191.

31. Atabay C, Tyutyunikov A, Scalise D, et al. Serum antibodies reactive with eye muscle membrane antigens are detected in patients with non-specific orbital inflammation. Ophthalmology. 1995;102(1): $145-153$. 
32. Porter JD, Khanna S, Kaminski HJ, et al. Extraocular muscle is defined by a fundamentally distinct gene expression profile. Proc Natl Acad Sci US A. 2001;98(21):12062-12067.

33. De Bellis A, Sanson D, Coronella C, et al. Serum antibodies to collagen XIII: a further good marker of active Graves' ophthalmopathy. Clin Endocrinol (Oxf). 2005;62(1):24-29.

34. Gopinath B, Musselman R, Beard N, et al. Antibodies targeting the calcium binding skeletal muscle protein calsequestrin are specific markers of ophthalmopathy and sensitive indicators of ocular myopathy in patients with Graves' disease. Clin Exp Immunol. 2006;145(1):56-62.

35. Gopinath B, Musselman R, Adams CL, Tani J, Beard N, Wall JR Study of serum antibodies against three eye muscle antigens and the connective tissue antigen collagen XIII in patients with Graves' disease with and without ophthalmopathy: correlation with clinical features. Thyroid. 2006;16(10):967-974.

36. Sandberg-Lall M, Hagg PO, Wahlstrom I, Pihlajaniemi T. Type XIII collagen is widely expressed in the adult and developing human eye and accentuated in the ciliary muscle, the optic nerve and the neural retina. Exp Eye Res. 2000;70(4):401-410.

37. Bartalena L, Bogazzi F, Pecori F, Martino E. Graves' disease occurring after subacute thyroiditis: report of a case and review of the literature. Thyroid. 1996;6(4):345-348.

38. Iitaka M, Momotani N, Hisaoka T, et al. TSH receptor antibodyassociated thyroid dysfunction following subacute thyroiditis. Clin Endocrinol (Oxf). 1998;48(4):445-453.

39. Amato G, Rotondi N, Salzano I, et al. Extraocular muscle antibodies positivity as the only serum marker of euthyroid Graves' ophthalmopathy following subacute thyroiditis: case report. J Clin Endocrinol Metab. 2000;85(3):950-952.

40. Bednarczuk T, Hiromatsu Y, Inoue Y, Yamamoto K, Wall JR, Nauman J. T-cell-mediated immunity in thyroid-associated ophthalmopathy. Thyroid. 2002;12(3):209-215.
41. Weetman AP, Cohen S, Gatter KC, Fells P, Shine B. Immunohistochemical analysis of the retrobulbar tissues in Graves' ophthalmopathy. Clin Exp Immunol. 1989;75(2):222-227.

42. Forster G, Otto E, Hansen C, Ochs K, Kahaly G. Analysis of orbital T cells in thyroid-associated ophthalmopathy. Clin Exp Immunol. 1998;112(3):427-434.

43. Pappa A, Lawson JM, Calder V, Fells P, Lightman S. T cells and fibroblasts in affected extraocular muscles in early and late thyroid associated ophthalmopathy. Br J Ophthalmol. 2000;84(5):517-522.

44. Nguyen B, Gopinath B, Tani J, Wescombe L, Wall JR. Peripheral blood T lymphocyte sensitisation against calsequestrin and flavoprotein in patients with Graves' ophthalmopathy. Autoimmunity. 2008;41(5):372-376.

45. Bednarczuk T, Gopinath B, Ploski R, Wall JR. Susceptibility genes in Graves' ophthalmopathy: searching for a needle in a haystack? Clin Endocrinol (Oxf). 2007;67(1):3-19.

46. Thornton J, Kelly SP, Harrison RA, Edwards R. Cigarette smoking and thyroid eye disease: a systematic review. Eye. 2007;21(9): 1135-1145.

47. Vestergaard P. Smoking and thyroid disorders - a meta-analysis. Eur J Endocrinol. 2002;146(2):153-161.

48. Wescombe L, Lahooti H, Gopinath B, Wall JR. The cardiac calsequestrin gene (CASQ2) is up-regulated in the thyroid in patients with Graves' ophthalmopathy - support for a role of autoimmunity against calsequestrin as the triggering event. Clin Endocrinol. 2010. In Press.

49. Diehn JJ, Diehn M, Marmor MF, Brown PO. Differential gene expression in anatomical compartments of the human eye. Genome Biol. 2005; 6(9):R74.

50. Gerding MN, van der Meer JW, Broenink M, Bakker O, Wiersinga WM, Prummel M. Association of thyrotrophin receptor antibodies with the clinical features of Graves' ophthalmopathy. Clin Endocrinol (Oxf). 2000;52(3):267-271.
Clinical Ophthalmology

\section{Publish your work in this journal}

Clinical Ophthalmology is an international, peer-reviewed journal covering all subspecialties within ophthalmology. Key topics include: Optometry; Visual science; Pharmacology and drug therapy in eye diseases; Basic Sciences; Primary and Secondary eye care; Patient Safety and Quality of Care Improvements. This journal is indexed on

\section{Dovepress}

PubMed Central and CAS, and is the official journal of The Society of Clinical Ophthalmology (SCO). The manuscript management system is completely online and includes a very quick and fair peer-review system, which is all easy to use. Visit http://www.dovepress.com/ testimonials.php to read real quotes from published authors. 